\title{
DESEMPENHO DE UM HIDROCICLONE CONCENTRADOR OTIMIZADO
}

\author{
M. T. V. SOUZA ${ }^{1}$, S. M. GONÇALVES ${ }^{1}$, L. G. M. VIEIRA ${ }^{1}$ e M. A. S. BARROZO ${ }^{1}$ \\ ${ }^{1}$ Universidade Federal de Uberlândia, Faculdade de Engenharia Química \\ E-mail para contato: sugoncalves.m@gmail.com
}

\begin{abstract}
RESUMO - Hidrociclones são separadores centrífugos usados tanto na clarificação quanto na concentração de suspensões. Em virtude da crescente demanda por redução de custos no setor industrial, uma nova geometria otimizada foi confeccionada para minimizar o número de Euler e, além de apresentar baixo gasto energético, apresentou características concentradoras e eficiências satisfatórias. Através de ensaios experimentais combinando diferentes diâmetros de underflow e quedas de pressão, as principais respostas relacionadas à hidrociclonagem foram determinadas, como razão de líquido, número de Euler e eficiência total. No nível mais alto das variáveis estudadas, ou seja, no diâmetro de underflow de $5 \mathrm{~mm}$ e queda de pressão de 1,77 bar, a razão de líquido alcançada foi cerca de 8\%, número de Euler de 912 e eficiência total de aproximadamente $68 \%$.
\end{abstract}

\section{INTRODUÇÃO}

Os hidrociclones são equipamentos centrífugos muito utilizados em diversas áreas do setor industrial para separações líquido-líquido ou sólido-líquido, visando à concentração de suspensões ou classificação de partículas por tamanho ou densidade. São equipamentos simples e de tamanho reduzido em relação a outros separadores, apresentando assim, baixo custo de aquisição, manutenção, operação (SVAROVSKY, 2000).

Os hidrociclones convencionais possuem uma região cilíndrica, por onde a suspensão é alimentada em uma entrada tangencial, bem como uma região cônica. A suspensão concentrada (underflow), constituída por partículas maiores, é direcionada por ação da força centrífuga às paredes do equipamento e então é coletada no orifício inferior da parte cônica. $\mathrm{Na}$ parte superior cilíndrica do hidrociclone há um tubo, chamado vortex finder, localizado axialmente por onde a suspensão diluída (overflow), dotada de partículas finas, é descarregada.

Vieira (2006) estudou a modalidade cônico-filtrante em diferentes configurações, concluindo que a geometria do equipamento deve ser escolhida dependendo do objetivo da demanada industrial (concentração ou classificação).

Com o objetivo de melhorar a separação sólido-líquido, Silva (2014) encontrou três novas geometrias de hidrociclones filtrantes através de técnicas de otimização combinando as superfícies de respostas com um algoritmo de Evolução Diferencial. Uma das configurações, além de ir de encontro ao objetivo inicial (minimização de custos energéticos), também 
apresentou características concentradoras, o que despertou o interesse de estudo desta configuração geométrica na modalidade convencional.

Este estudo teve como objetivo principal a minimização de custos energéticos e manutenção da eficiência de separação do hidrociclone concentrador estudado por Silva (2014), mas em sua modalidade convencional. A este hidrociclone convencional foi the dada a denominação de HCO. Para tanto, o desempenho do hidrociclone HCO foi estudado mediante a manipulação das dimensões do orifício de underflow e variações na queda de pressão do hidrociclone. Neste tema, existem alguns conceitos básicos envolvidos na temática dos hidrociclones que são fundamentais para melhor entendimento deste trabalho, tais como a razão de líquido, a eficiência total e o número de Euler, doravante especificados.

\subsection{Razão de Líquido}

A razão de líquido representa a fração de líquido que, inicialmente alimentada no hidrociclone, é coletada na corrente de underflow, estando diretamente relacionada com o poder de classificação do equipamento. Está representada através da Equação 1 e pode ser facilmente determinada uma vez conhecidas as vazões volumétricas das correntes de alimentação $\left(\mathrm{Q}_{\mathrm{A}}\right)$ e underflow $\left(\mathrm{Q}_{\mathrm{U}}\right)$ com suas respectivas concentrações volumétricas.

$$
\mathrm{Rl}=\frac{\mathrm{Q}_{\mathrm{U}}\left(1-\mathrm{C}_{\mathrm{VU}}\right)}{\mathrm{Q}_{\mathrm{A}}\left(1-\mathrm{C}_{\mathrm{VA}}\right)}
$$

O cálculo das concentrações volumétricas das correntes pode ser visualizado através da Equação 2, onde i representa uma corrente genérica (alimentação, underflow ou overflow).

$$
\mathrm{C}_{\mathrm{Vi}}=\frac{1}{\left[\frac{\rho_{\mathrm{S}}}{\rho}\left(\frac{1}{\mathrm{C}_{\mathrm{Wi}}}-1\right)+1\right]}
$$

\subsection{Eficiência Total}

O percentual de massa de sólidos coletado na corrente de underflow frente à quantidade inicialmente introduzida na alimentação de cada hidrociclone é contabilizado pela eficiência total do hidrociclone. Esta resposta pode ser obtida através do conhecimento das vazões mássicas das correntes de alimentação e underflow, e, das suas respectivas concentrações mássicas, a eficiência está representada através da Equação 3.

$$
\eta=\frac{\mathrm{C}_{\mathrm{wU}} \mathrm{W}_{\mathrm{U}}}{\mathrm{C}_{\mathrm{wA}} \mathrm{W}_{\mathrm{A}}}
$$

\subsection{Número de Euler}

O número de Euler é uma grandeza que representa a capacidade de operação, ou seja, o consumo energético do equipamento através da razão entre a queda de pressão pela energia cinética por unidade de volume na parte cilíndrica. A Equação 4 fornece as informações necessárias para o cálculo do número de Euler quando conhecidas as vazões volumétricas de 


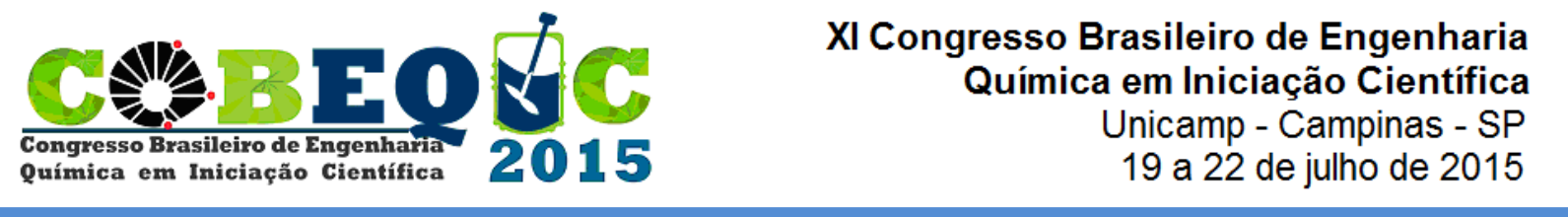

alimentação, queda de pressão e densidade da água na temperatura de operação em cada configuração concebida para o hidrociclone HCO.

$$
\mathrm{Eu}=\frac{-\Delta \mathrm{P}}{\frac{\rho}{2}\left(\frac{4 \mathrm{Q}}{\pi \mathrm{D}_{\mathrm{C}}^{2}}\right)^{2}}
$$

\section{MATERIAIS E MÉTODOS}

\subsection{Unidade Experimental}

A unidade experimental encontra-se no Laboratório de Separação e Energias Renováveis (LASER) da Faculdade de Engenharia Química da UFU, dotada dos principais acessórios para reproduzir as mesmas condições industriais durante a hidrociclonagem (reservatório, agitador mecânico, bomba centrífuga, flowmeter, manômetro digital etc.).

As dimensões geométricas do hidrociclone concentrador otimizado (HCO) estão mostradas na Figura 1.

Figura 1 - Dimensões Geométricas do Hidrociclone Concentrador Otimizado em milímetros

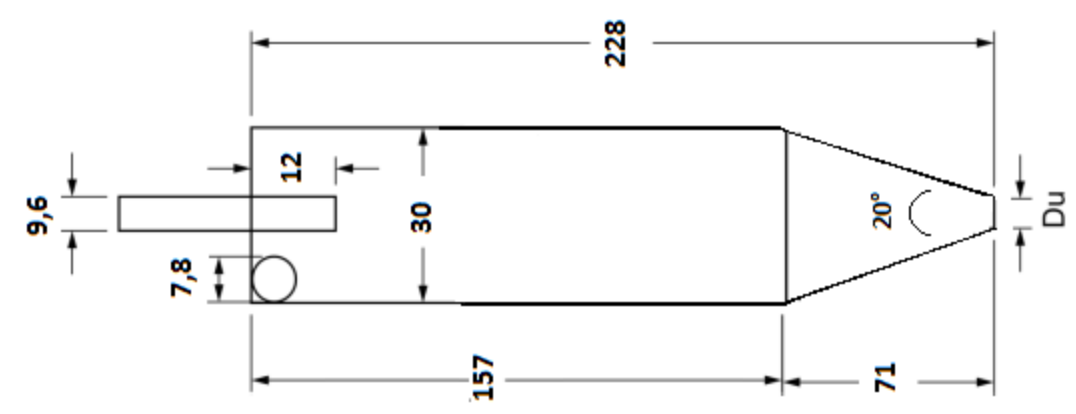

\subsection{Material Utilizado}

Para os ensaios experimentais foi utilizado quartzito, material particulado cedido pela ANEX Mineração, sediada em Itabirito (MG). Este material particulado é composto predominantemente por silício $\left(\mathrm{SiO}_{2}\right)$, apresenta densidade média de $2,69 \mathrm{~g} / \mathrm{cm}^{3}$ e distribuição granulométrica regida pelo modelo RRB (Rosin-Rammlet-Bennet), com os parâmetros $\mathrm{d}_{63,2}=$ $12,45 \mu \mathrm{m}$ e $\mathrm{n}=0,91$.

\subsection{Metodologia}

$\mathrm{Na}$ montagem do equipamento foram utilizadas as dimensões características descritas na Figura 1 combinadas com os diâmetros de underflow de 3, 4 e $5 \mathrm{~mm}$. A suspensão aquosa do material particulado (quartzito), a 1\% em volume, foi introduzida no tanque e para cada configuração do hidrociclone (HCO) foram combinados quatro níveis de queda de pressão: $0,88,1,18,1,47$ e 1,77 bar. 
As concentrações mássicas das principais correntes do hidrociclone foram determinadas por técnicas de gravimetria. As distribuições granulométricas das principais correntes do hidrociclone foram determinadas por difração a laser no Mastersizer da Malvern. As vazões mássicas das principais correntes do hidrociclone foram determinadas pelo medidor eletromagnético do tipo Coriolis.

\section{RESULTADOS E DISCUSSÃO}

Os resultados experimentais para a razão de líquido do $\mathrm{HCO}$ em diferentes quedas de pressão e diâmetros de underflow estão apresentados na Figura 2.

Figura 2 - Razão de líquido em função da queda de pressão e diâmetro de underflow

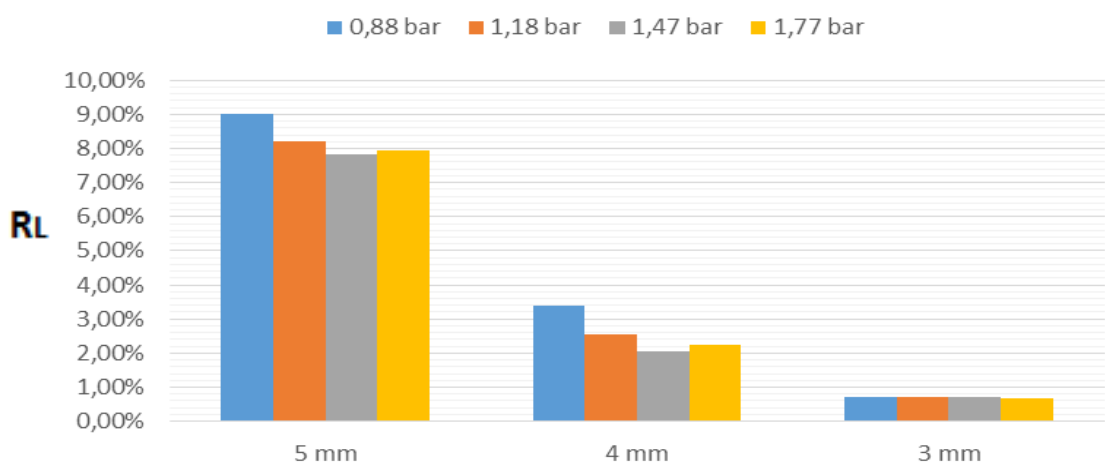

Observa-se que para o menor diâmetro de underflow $(3 \mathrm{~mm})$ as razões de líquido se mantiveram num mesmo patamar, cerca de $0,7 \%$. Para o diâmetro de 4 e $5 \mathrm{~mm}$, a variação da queda de pressão levou a flutuação dos valores em aproximadamente $1 \%$. O valor máximo para $\mathrm{R}_{\mathrm{L}}$ foi de $9 \%$ para a maior queda de pressão e maior diâmetro de underflow. Os dados experimentais confirmam o poder concentrador deste hidrociclone mesmo. Na Figura 3 é mostrado o efeito das variáveis estudadas no Número de Euler.

Figura 3 - Número de Euler em função da queda de pressão e diâmetro de underflow.

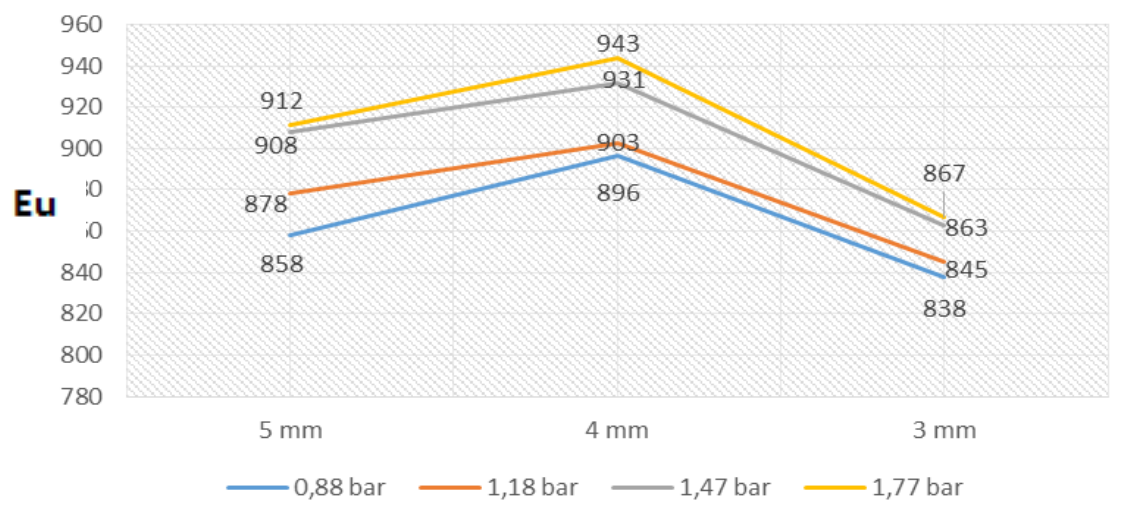

A linha azul reafirma que quanto menor a perda de carga, menor o consumo energético e menor o número de Euler. $\mathrm{O}$ aumento da pressão, mantendo a variável diâmetro de underflow constante, aumenta o consumo energético e a capacidade de processamento. 
Os dados experimentais, numa mesma queda de pressão, indicaram menores números de Euler para o menor diâmetro de underflow estudado $(3 \mathrm{~mm}$ ), ao invés do maior orifício (5 $\mathrm{mm}$ ). Ordinariamente era esperado que, quanto maior fosse a restrição no orifício de underflow maior seria o número de Euler. Entretanto, para este separador, em específico, observou-se um comportamento anômalo, indicando que o Número de Euler do hidrociclone HCO não foi influenciado apenas pelo efeito isolado do diâmetro de underflow. Certamente, houve efeitos de outras dimensões geométricas do separador que conjuntamente com o diâmetro de underflow proporcionaram este comportamento atípico. Dentre elas, acredita-se que o comprimento do vortex finder $(\ell)$ e o comprimento da parte cilíndrica do hidrociclone (L) sejam os principais responsáveis por esta anomalia (combinação de feitos), haja vista que o primeiro permaneceu em uma pequena dimensão $(12 \mathrm{~mm})$, enquanto o segundo foi utilizado em uma dimensão relativamente grande $(157 \mathrm{~mm})$.

Na Figura 4 são ilustrados os efeitos estudados na resposta da Eficiência total do hidrocione HCO.

Figura 4 - Eficiência total do hidrociclone HCO em função da queda de pressão e diâmetro de underflow

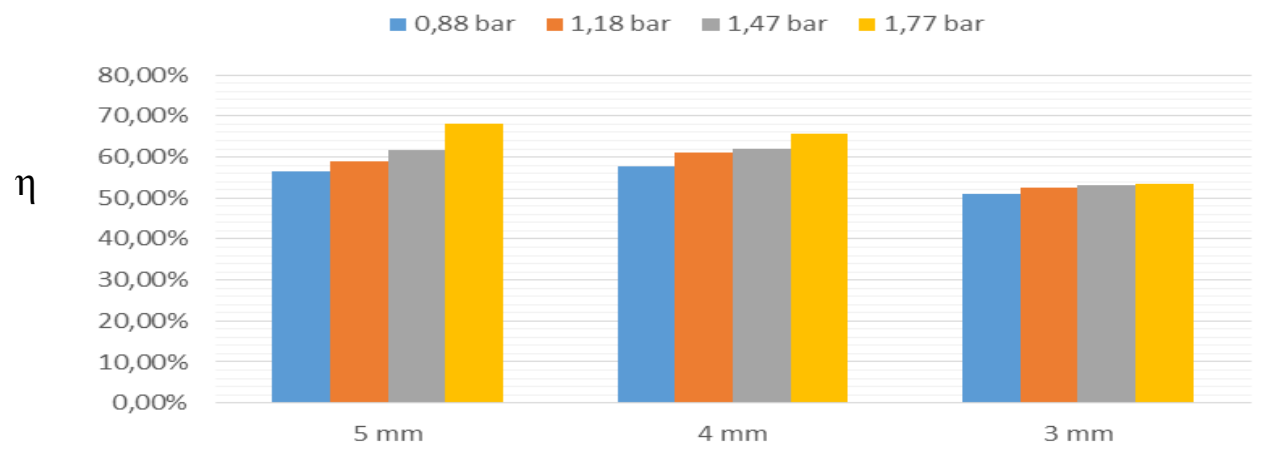

A variação da eficiência do hidrociclone (HCO) foi crescente em relação ao aumento da queda de pressão, e decrescente em relação ao diâmetro de underflow. Quanto menor a restrição ao escoamento menor foi a quantidade de material coletada e, portanto, menor foi a eficiência total. Pôde-se observar que mesmo a eficiência atingindo patamares de $50 \%$, este comportamento ainda se mostrou satisfatório, haja vista que nestas condições $\left(D_{U}=3 \mathrm{~mm}\right)$ houve uma excelente concentração de sólidos, haja vista que as respectivas Razões de Líquido foram menores que 1\% (Figura 2).

A melhor eficiência $(68,19 \%)$ foi observada no hidrociclone dotado do maior diâmetro de underflow na maior queda de pressão de operação (1,77 bar). Em suma, essa condição operacional poderia ser eleita como a melhor dentre todas porque conciliou eficiências satisfatórias com baixo gasto energético $(\mathrm{Eu}=912)$ e pequenas razões de líquido $(9 \%)$. Se o principal objetivo fosse concentrar a corrente de alimentação, a condição de Du de $3 \mathrm{~mm}$ e queda de pressão de 0,88 bar apresentaria o menor gasto energético $(\mathrm{Eu}=838)$.

\section{CONCLUSÕES}

Através dos dados experimentais apresentados foi possível notar que o número de Euler permaneceu em níveis aceitáveis, mesmo na menor restrição do diâmetro de underflow 


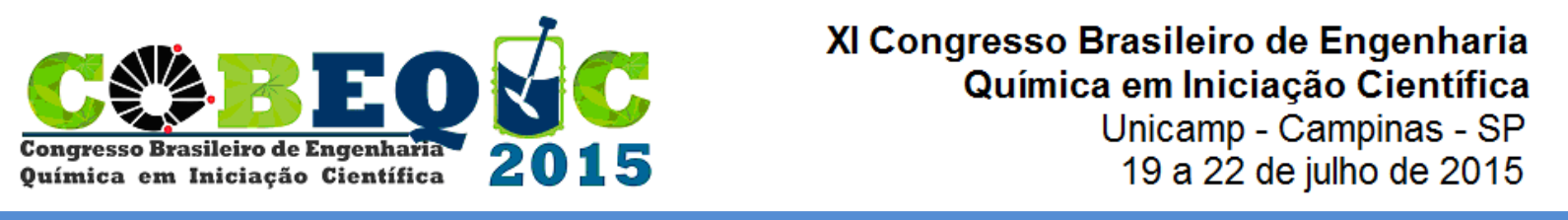

( $3 \mathrm{~mm}$ ). Foi possível concluir ainda que a diminuição do diâmetro de underflow proporcionou um interessante efeito concentrador da suspensão. Neste mesmo aspecto, a eficiência total não foi prejudicada, que também permaneceu em níveis aceitáveis com a variação do diâmetro de underflow. Logo, a depender das necessidades técnicas do processo (concentração ou separação), o hidrociclone HCO pode ser um equipamento versátil mediante a manipulação da dimensão do orifício de underflow.

\section{NOMENCLATURA}

$\mathrm{C}_{\mathrm{VA}}$ - concentração volumétrica de sólidos na corrente de alimentação (-)

$\mathrm{C}_{\mathrm{VU}}$ - concentração volumétrica de sólidos na corrente de underflow (-)

$\mathrm{C}_{\mathrm{WA}}$ - concentração mássica de sólidos na corrente de alimentação (-)

$\mathrm{C}_{\mathrm{WU}}$ - concentração mássica de sólidos na corrente de underflow (-)

$\mathrm{D}_{\mathrm{C}}$ - diâmetro da parte cilíndrica do hidrociclone (L)

$\mathrm{Eu}$ - número de Euler (-)

$\eta$ - eficiência total (-)

$\mathrm{Q}_{\mathrm{A}}$ - vazão volumétrica da corrente de alimentação $\left(\mathrm{L}^{3} \mathrm{~T}^{-1}\right)$

$\mathrm{Q}_{\mathrm{U}}$ - vazão volumétrica da corrente de underflow $\left(\mathrm{L}^{3} \mathrm{~T}^{-1}\right)$

$\mathrm{R}_{\mathrm{L}}$ - razão de líquido (-)

$\mathrm{W}_{\mathrm{A}}$ - vazão mássica da corrente de alimentação $\left(\mathrm{MT}^{-1}\right)$

$\mathrm{W}_{\mathrm{U}}$ - vazão mássica da corrente de underflow $\left(\mathrm{MT}^{-1}\right)$

$-\Delta \mathrm{P}$ - queda de pressão no hidrociclone $\left(\mathrm{ML}^{-1} \mathrm{~T}^{-2}\right)$

$\rho$ - densidade do fluido $\left(\mathrm{ML}^{-3}\right)$

$\rho_{\mathrm{S}}$ - densidade do sólido/material particulado $\left(\mathrm{ML}^{-3}\right)$

\section{REFERÊNCIAS}

PRICE, K.V.; STORN, R.M.; LAMPINEN, J.A. Differential Evolution: A Practical Approach to Global Optimization. Natural Computing Series: Springer Verlag, 2005.

SILVA, N. K. G. Estudo da Otimização da Configuração Do Hidrociclone Filtrante. Dissertação (Mestrado em Engenharia Química) - UFU, Uberlândia, 2014.

SVAROVSKY, L. Solid-Liquid Separation. 554 p. 4th Edition. Linacre House, Jordan Hill, Oxford OX2 8DP: Butterworth-Heinemann, 2000.

VIEIRA, L. G. M. Otimização dos Processos de Separação em Hidrociclones Filtrantes. 2006. 297 f. Tese (Doutorado em Engenharia Química) - UFU, Uberlândia, 2006.

\section{AGRADECIMENTOS}

Os autores agradecem à Capes e ao CNPq, como órgãos de fomento da pesquisa e ao Laboratório de Separação e Energias Renováveis (LASER) da Faculdade de Engenharia Química da Universidade Federal de Uberlândia pelo suporte na execução deste trabalho. 